$01,11,12$

\title{
Моделирование размерных эффектов при фазовых превращениях в субмикронных частицах сплава $\mathrm{Au}-\mathrm{Pt}-\mathrm{Pd}$
}

\author{
(C) В.Б. Федосеев \\ Институт металлоорганической химии им. Г.А. Разуваева РАН, \\ Нижний Новгород, Россия \\ E-mail: vbfedoseev@yandex.ru
}

Поступила в Редакцию 15 мая 2021 г.

В окончательной редакции 15 мая 2021 г.

Принята к публикации 22 мая 2021 г.

\begin{abstract}
Размерные эффекты наблюдаются при фазовых равновесиях в микро- и наночастицах. На фазовых диаграммах они отображаются в виде сдвига характеристических линий и точек. Рассмотрено моделирование и графическое отображение этих эффектов для тройных систем. На примере твердого раствора $\mathrm{Au}-\mathrm{Pt}-\mathrm{Pd}$ методами химической термодинамики смоделировано влияние состава сплава на область существования состояний ядро-оболочка (core-shell) для частиц радиусом $250 \mathrm{~nm}$. Показано уменьшение области расслаивания этого раствора и ее разделение за счет конкуренции состояний ядро-оболочка с сегрегацией $\mathrm{Pt}$ в core либо в shell фазе. На фазовой диаграмме построены коноды стабильных и метастабильных равновесных состояний ядро-оболочка. Приведены характеристики этих состояний (состав сосуществующих растворов, радиус core фазы). Описанные закономерности актуальны при рассмотрении каталитической активности частиц сплава $\mathrm{Au}-\mathrm{Pt}-\mathrm{Pd}$.
\end{abstract}

Ключевые слова: расслаивание раствора, тройная система, структура ядро-оболочка, метастабильные состояния.

DOI: 10.21883 /FTT.2021.10.51391.115

\section{1. Введение}

Размерные эффекты при фазовых превращениях проявляются на диаграммах состояния в виде заметных смещений характеристических точек и линий $[1,2]$, в существенных изменениях взаимной растворимости компонентов [3] и появлении метастабильных фаз [4]. Это приводит к тому, что огромный массив справочных данных о диаграммах состояния макросистем перестает быть надежным инструментом при работе с микрои нанообъектами. Особенности фазовых превращений в малом объеме важны при производстве и эксплуатации порошковых композиционных, ультрамелкозернистых материалов. Для бинарных смесей размерные эффекты при фазовых превращениях описаны во многих работах $[1-3,5]$. Естественно ожидать более сложного поведения от тройных систем, однако, отображение размерных эффектов на фазовых диаграммах для них в литературе практически отсутствуют [6].

В настоящей работе представлены фазовые диаграммы, отображающие размерные эффекты при фазовых превращениях трехкомпонентного сплава $\mathrm{Au}-\mathrm{Pt}-\mathrm{Pd}$, который может быть описан уравнениями состояния регулярного раствора [7]. Интерес к подобным сплавам связан с их каталитической активностью в процессах восстановлении кислорода и ,зеленой химии“ $[8-10]$. Они находят применение в качестве электрокатализаторов для топливных ячеек [11-13]. В большинстве случаев подобные сплавы используются в виде наночастиц и тонких пленок. При этом для частиц характерно образование структур типа ядро-оболочка (core-shell) [14,15], а для микрокристаллических пленок наблюдается сегрегация $\mathrm{Au}$ и $\mathrm{Pd}$ к границам зерна [16]. Как образование структурыя дро-оболочка, так и зернограничная сегрегация являются следствиями эволюции реального раствора в условиях ограниченного объема. При этом размерные эффекты при фазовых превращениях представляют особый интерес для интерпретации наблюдаемых явлений при оптимизации состава и свойств катализаторов и решении проблемы стабильности каталитических свойств этих материалов, которые связаны с самопроизвольной перестройкой структуры [17].

Задачей настоящей работы является моделирование размерных эффектов при фазовых превращениях в трехкомпонентном растворе на примере сплава $\mathrm{Au}-\mathrm{Pt}-\mathrm{Pd}$. Ранее нами была описана термодинамическая модель образования структуры ядро-оболочка для бинарного раствора $\mathrm{Pt}-\mathrm{Au}$, в котором каталитическая активность также определяется составом и удельной поверхностью shell фазы [18].

\section{2. Термодинамическая модель}

Для расчетов нами была использована термодинамическая модель $[3,4]$. От других методов расчетов фазовых равновесий $[19,20]$ ее отличает поиск всех минимумов энергии Гиббса гетерогенного состояния. Детальный анализ функции Гиббса воспроизводит не 
только глобальное равновесное состояние [7,21], но и метастабильные состояния, которые характерны для систем малого объема.

Функция Гиббса системы $G$ складывается из энергии Гиббса сосуществующих фаз и энергии межфазных границ

$$
G=\frac{n_{c} G_{c}+n_{s} G_{s}+\sigma_{s} A_{s}+\sigma_{s} A_{s}+\sigma_{c s} A_{c s}}{n_{c}+n_{s}},
$$

где $n_{j}$ - число молей в фазах $j=c, s$ (core и shell соответственно), $\sigma_{s}$ и $\sigma_{c s}-$ поверхностные натяжения на внешней (shell) и внутренней (core-shell) границах раздела фаз, вычисленные с использованием линейной аппроксимации $\sigma_{s}=\sum x_{i s} \sigma_{i}, \quad \sigma_{c s}=0.5 \sum\left(x_{i c}+x_{i s}\right) \sigma_{i}$ на основе свойств индивидуальных компонентов $\sigma_{i}$, $i=1,2,3$ - номер компонента (соответственно $\mathrm{Au}, \mathrm{Pt}$, $\mathrm{Pd}), x_{i j}=n_{i j} / \sum n_{i j}$ и $n_{i j}-$ мольная доля и число молей компонента $i$ в фазе $j, A_{c}, A_{s}$ - площадь поверхности core и shell фаз, $G_{j}-$ энергия Гиббса реального раствора соответствующей фазы.

Согласно [7] тройная система $\mathrm{Au}-\mathrm{Pt}-\mathrm{Pd}$ в твердом состоянии имеет единственную фазу, а именно расслаивающийся твердый раствор. Его энергия (при $T=1073 \mathrm{~K}, \mathrm{cal} / \mathrm{mol}$ ) описывается уравнением регулярного раствора $[7,21]$

$$
\begin{aligned}
G= & x_{1} x_{2} x_{3} 3036-x_{1} x_{3}\left(11141 x_{1}-3065 x_{3}\right) \\
& -x_{2} x_{3}\left(5234 x_{3}+3021 x_{2}\right)-x_{1} x_{2}\left(634 x_{1}-5691 x_{2}\right) \\
& -T\left(\sum x_{i} \ln x_{1}-3 x_{1} x_{3}+2.9 x_{3}^{2} x_{2}+1.7 x_{3} x_{2}^{2}\right. \\
& \left.+0.9 x_{1} x_{2}^{2}+3.5 x_{1}^{2} x_{2}-9 x_{1} x_{2} x_{3}\right) .
\end{aligned}
$$

Предполагается, что обе фазы (ядро и оболочка) имеют сферическую форму. В этом случае условия сохранения количества вещества однозначно связывают объем фаз $V_{j}=n_{1 j} V_{1}+n_{2 j} V_{2}+n_{3 j} V_{3}$ (где $V_{i}$ - мольные объемы компонентов), радиусы $\left(r_{j}\right)$ и площади внешней и межфазной границ $\left(A_{c}, A_{s}\right)$, концентрации компонентов в сосуществующих фазах $\left(x_{i j}\right)$ и число молей компонентов $n_{i j}$ :

$$
\begin{gathered}
r_{c}=\left(\frac{3}{4 \pi} V_{c}\right)^{1 / 3}, \quad A_{c}=4 \pi r_{c}^{2}, \\
r_{s}=\left(\frac{3}{4 \pi}\left(V_{c}+V_{s}\right)\right)^{1 / 3}, \quad A_{s}=4 \pi r_{s}^{2} .
\end{gathered}
$$

Подобные (2) соотношения при необходимости можно сформулировать для межфазных границ более сложной формы $[2,22]$.

Если задан исходный состав системы $n_{i}$, то $n_{i}=n_{i c}+n_{i s}$ и энергия Гиббса (1) становится функцией трех независимых переменных $G\left(n_{i c}\right)$. Минимумы этой функции соответствуют равновесным состояниям системы и полностью определяют состав и объем сосуществующих фаз. Для удобства при моделировании использована энергия фазового перехода $\Delta_{t r} G\left(n_{i c}\right)=G\left(n_{i c}\right)-G(0)$, позволяющая сразу оценить стабильность или метастабильность гетерогенных состояний относительно гомогенного раствора.

\section{3. Результаты моделирования}

Необходимые для расчета мольные объемы $\mathrm{Au}, \mathrm{Pt}$ и $\mathrm{Pd}$ приводятся в справочной литературе и базах данных, значения поверхностной энергии заимствованы из обзора [23, table 68].

Для поиска минимумов использовался трехмерный массив значений $\Delta_{t r} G\left(n_{i c}\right)$, соответствующих переносу в core фазу разного количества компонентов $0 \leq n_{i c} \leq n_{i}$. Соответствующие минимумам значения $n_{i c}$ определяют радиус core фазы и состав сосуществующих растворов. Шаг $\Delta n_{i c}$ определял локализацию равновесных состояний с точностью до $1.5-2 \%$.

Энергия Гиббса состояния ядро-оболочка имеет, как правило, не менее двух минимумов, соответствующих разному размещению сосуществующих растворов в core и shell положениях. Чем меныше объем частиц, тем сильнее эти состояния отличаются составом фаз и термодинамическими характеристиками $[3,4]$. Чаще всего наиболее выгодным является состояние, при котором поверхностная энергия core фазы превышает поверхностную энергию shell фазы.

Сопоставление состояний ядро-оболочка частицы радиусом $250 \mathrm{~nm}$ и макросистемы приведено на рис. 1.

На рис. 1 показаны коноды (линии, соединяющие сосуществующие фазы) для составов с $x_{\mathrm{Pd}}=0.325,0.25$, $0.175,0.125,0.075,0.025$ и $x_{\mathrm{Au}} / x_{\mathrm{Pt}}=0.65$ на фоне диаграммы расслаивания сплава $\mathrm{Au}-\mathrm{Pt}-\mathrm{Pd}$ при $1073 \mathrm{~K}$. Диаграмма демонстрирует несколько особенностей состояний ядро-оболочка.

Прежде всего, существуют две области термодинамически выгодных состояний $\left(\Delta_{t r} G<0\right)$. Более крупная область соответствует состояниям с высокой концентрацией Pt в core фазе, в другой концентрация Pt выше в shell фазе. При увеличении размера частицы области эти совпадают с областью гетерогенности макросистемы, а при уменьшении сжимаются и пропадают. В области их пересечения оба состояния выгодны и могут конкурировать между собой, если имеют сопоставимую энергию образования.

На рис. 1 приведены коноды для составов с $x_{\mathrm{Au}} / x_{\mathrm{Pt}}=0.65$. Стабильные состояния изображены сплошными прямыми, метастабильные - штриховыми. Отклонение концов конод от бинодали макросистемы свидетельствует о том, что даже для стабильных состояний состав сосуществующих растворов существенно отличается от состояния макросистемы.

В таблице приведены характеристики для двух состояний ядро-оболочка, изображенных на рис. 1.

При $x_{\mathrm{Pd}}=0.325$ (рис. 1) состав сплава находится за пределами области гетерогенности макросистемы и существование метастабильных состояний в этой области 
Состояния ядро-оболочка частицы радиусом $250 \mathrm{~nm}$

\begin{tabular}{|c|c|c|c|c|c|c|c|c|}
\hline \multirow{2}{*}{ № } & \multirow{2}{*}{$\begin{array}{l}r_{c}, \\
\mathrm{~nm}\end{array}$} & \multirow{2}{*}{$\begin{array}{l}\Delta_{t r} G, \\
\mathrm{~kJ} / \mathrm{mol}\end{array}$} & \multicolumn{3}{|c|}{ Ядро } & \multicolumn{3}{|c|}{ Оболочка } \\
\hline & & & $x_{1}$ & $x_{2}$ & $x_{3}$ & $x_{1}$ & $x_{2}$ & $x_{3}$ \\
\hline \multicolumn{9}{|c|}{$x_{\mathrm{Pd}}=0.175, \quad x_{\mathrm{Au}} / x_{\mathrm{Pt}}=0.65$} \\
\hline 1 & $\mid 157$ & 8.2 & 0.0592 & 0.8243 & 0.1166 & 0.3667 & 0.4385 & $\mid 0.1948$ \\
\hline 2 & 167 & 8.3 & 0.0613 & 0.8198 & 0.1189 & 0.389 & 0.4113 & 0.1997 \\
\hline 3 & 170 & 8.2 & 0.0582 & 0.8218 & 0.12 & 0.3982 & 0.4006 & 0.2011 \\
\hline 4 & 178 & 8.2 & 0.0612 & 0.815 & 0.1237 & 0.4209 & 0.3743 & 0.2048 \\
\hline 5 & 181 & 8.2 & 0.0674 & 0.8043 & 0.1283 & 0.4301 & 0.3651 & 0.2048 \\
\hline 6 & 246 & 30.8 & 0.2712 & 0.5548 & 0.1739 & 0.6514 & 0.1512 & 0.1974 \\
\hline \multicolumn{9}{|c|}{$x_{\mathrm{Pd}}=0.075, \quad x_{\mathrm{Au}} / x_{\mathrm{Pt}}=0.65$} \\
\hline 7 & 200 & -23.4 & 0.0484 & 0.9142 & 0.0374 & 0.6233 & 0.2608 & $\mid 0.1159$ \\
\hline 8 & 126 & 5.1 & 0.6454 & 0.2523 & 0.1023 & 0.2783 & 0.6505 & 0.0711 \\
\hline 9 & 129 & 5.1 & 0.6631 & 0.2345 & 0.1024 & 0.2716 & 0.6576 & 0.0708 \\
\hline 10 & 192 & -13.2 & 0.6527 & 0.2317 & 0.1156 & 0.0673 & 0.8893 & 0.0433 \\
\hline \multicolumn{9}{|c|}{$x_{\mathrm{Pd}}=0.075, \quad x_{\mathrm{Au}} / x_{\mathrm{Pt}}=0.75$} \\
\hline 11 & 219 & -12.0 & 0.0529 & 0.8986 & 0.0486 & 0.6135 & 0.2548 & 0.1316 \\
\hline 12 & 168 & -13.3 & 0.6247 & 0.2458 & 0.1295 & 0.0680 & 0.8796 & 0.0524 \\
\hline
\end{tabular}

заслуживает особого внимания. Эти состояния имеют сопоставимые по величине энергии $\left(\Delta_{t r} G \approx 32 \mathrm{~kJ} / \mathrm{mol}\right)$ и большой объем ядра (до $99 \mathrm{vol} . \%$ ). Более выгодными из них являются состояния с полным отсутствием Pt в shell фазе. Они пропадают при увеличении содержания $\mathrm{Pd}$ или $\mathrm{Au}$. Низкое содержание Pt в shell фазе характерно для всех состояний с $x_{\mathrm{Pd}}=0.325,0.25$ и $x_{\mathrm{Au}} / x_{\mathrm{Pt}}=0.65$, показанных на рис. 1.

Все равновесные состояния при $x_{\mathrm{Pd}}=0.175$ (таблица, рис. 1) метастабильны. Состояния 1-5 имеют сопоставимые величины и состав сосуществующих фаз. Core фаза имеет высокую концентрацию $\mathrm{Pt}$ и ее объем не превышает $40 \mathrm{vol} . \%$. Состояние 6 отличается высокой энергией образования $\Delta_{t r} G$, большим объемом ядра (95 vol.\%), меньшей концентрацией Pt и в core, и в shell фазе.

При $x_{\mathrm{Pd}}=0.125$ состав частицы попадает в одну из областей термодинамически выгодных состояний (рис. 1) и имеет одно стабильное и одно метастабильное состояние.

При $x_{\mathrm{Pd}}=0.075$ частица имеет два термодинамически выгодных состояния. Это парные состояния 7 и 10 (таблица и рис. 1), отличающиеся положением сосуществующих фаз в структуре ядро-оболочка. Глобальному равновесному состоянию соответствует оболочка с низкой концентрацией Pt. Метастабильные состояния 8 и 9 сопоставимы по составу фаз с состоянием 10, но имеют небольшой размер ядра $(\sim 13 \mathrm{vol} . \%)$. При большей доле $\mathrm{Au}$ и той же концентрации $\mathrm{Pd}$ (таблица, состояния 11,12 ) глобальному равновесному состоянию соответствует оболочка с высокой концентрацией $\mathrm{Pt}$. Парные состояния 7,10 и 11,12 являются примером конкуренции и бистабильности фазовых равновесий.

Другой размерный эффект демонстрирует зависимость энергии образования состояний ядро-оболочка от радиуса частицы состава $x_{\mathrm{Pd}}=0.075, x_{\mathrm{Au}} / x_{\mathrm{Pt}}=0.65$ (рис. 2). С уменьшением радиуса частиц меняется не только энергия, но и число метастабильных состояний.

Макросистема имеет два неразличимых по энергии гетерогенных состояния. Для микронных и субмикронных частиц становится существенным вклад поверхностной энергии, поэтому энергия этих состояний отличается и зависит от размера частицы и положения сосуществующих растворов в структуре ядро-оболочка. При этих размерах появляются дополнительные метастабильные состояния. С уменьшением размера $(r<110 \mathrm{~nm})$ частицы имеют только метастабильные состояния.

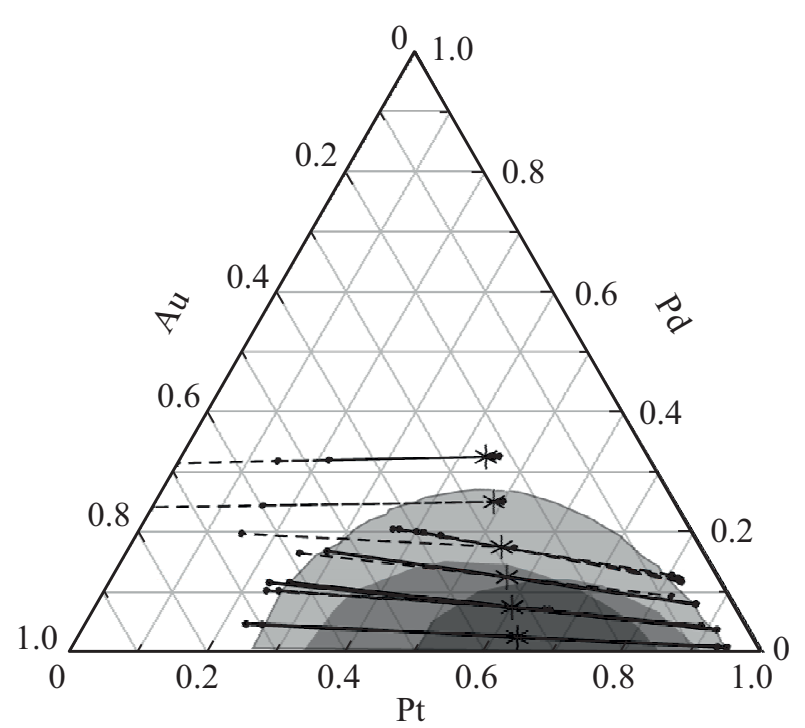

Рис. 1. Состояния ядро-оболочка частиц радиусом $250 \mathrm{~nm}$. Область гетерогенности макросистемы выделена светло-серым цветом, две перекрывающиеся области с $\Delta_{t r} G<0-$ темносерым. Коноды состояний с $\Delta_{t r} G<0$ показаны сплошными линиями, с $\Delta_{t r} G>0-$ штриховыми, соответствующий им состав помечен звездочками.

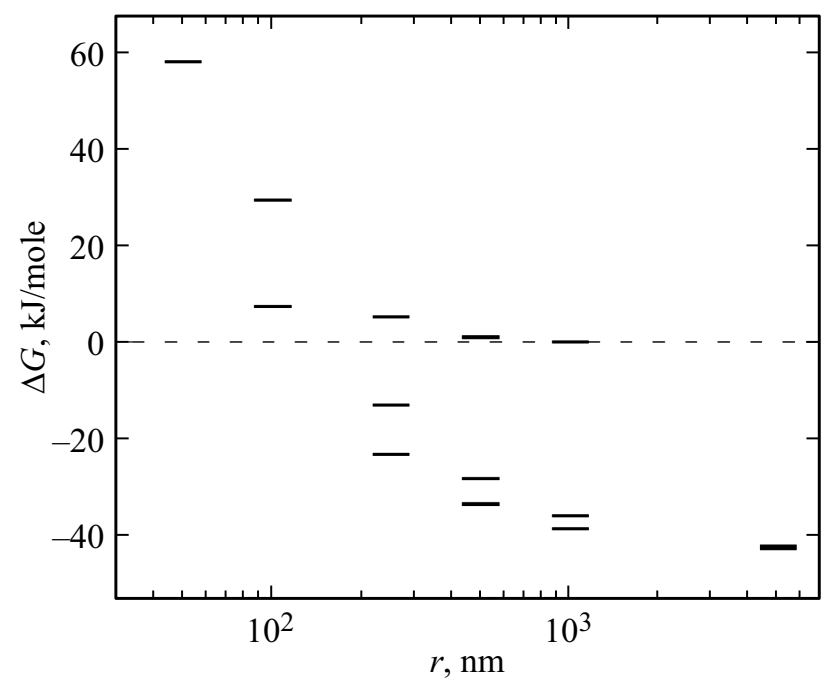

Рис. 2. Зависимость энергии образования состояний ядрооболочка от радиуса частицы при $x_{\mathrm{Pd}}=0.075, x_{\mathrm{Au}} / x_{\mathrm{Pt}}=0.65$. 
Эта размерная зависимость воспроизводится и для других составов частицы. Состав определяет количество термодинамически стабильных и метастабильных состояний, а также интервалы размеров, в которых эти состояния существуют.

\section{4. Заключение}

В общем случае размер и состав частицы опре деляет количество равновесных состояний, энергию образования $\left(\Delta_{t r} G\right)$, структуру $\left(r_{c}\right)$ и состав сосуществующих растворов $\left(x_{i j}\right)$.

Моделирование частиц сплава $\mathrm{Pt}-\mathrm{Pd}-\mathrm{Au}$ показывает, что они могут иметь устойчивые состояния ядрооболочка с высоким содержанием $\mathrm{Au}$ или $\mathrm{Pd}$ как в core, так и в shell фазах. Имея сопоставимые энергии образования, эти состояния могут конкурировать между собой, что подтверждается одновременным образованием при синтезе частиц со структурой $\mathrm{Au}_{\text {shell }}-\mathrm{Pt}_{\text {core }}$ и $\mathrm{Pt}_{\text {shell }}-\mathrm{Au}_{\text {core, }}$ или $\mathrm{Pd}_{\text {shell }}-\mathrm{Pt}_{\text {core }}$ и $\mathrm{Pd}_{\text {core }}-\mathrm{Pt}_{\text {shell }}[10,24]$. Число метастабильных состояний возрастает с увеличением содержания $\mathrm{Pd}$ или приближением к границам гетерогенности макросистемы.

Множество метастабильных состояний создает проблему, связанную с деградацией структуры ядрооболочка и свойств катализатора. В [17] описана эволюция частиц, при которой shell фаза обогащается $\mathrm{Pt}$, после чего частица переходит в стабильное состояние гомогенного раствора $\mathrm{Pt}-\mathrm{Pd}-\mathrm{Au}$. Подобные эффекты могут возникать при высоком содержании $\mathrm{Pd}$ (см. рис. $\left.1, x_{\mathrm{Pd}}=0.325,0.25,0.175\right)$. Решением данной проблемы, согласно рис. 1, может быть переход в область термодинамически устойчивых $\left(\Delta_{t r} G<0\right)$ coreshell-состояний, например, за счет уменьшения концентрации Pd в сплаве.

Высокие каталитические свойства частиц с оболочкой из $\mathrm{Pd}$ или $\mathrm{Pd}-\mathrm{Au}$ отмечены в $[14,15]$. Немонотонная зависимость каталитической активности от состава оболочки [17] делает актуальной оптимизацию составов и дисперсности подобных сплавов для получения частиц с устойчивой структурой ядро-оболочка.

Описанные размерные эффекты существенно меняют условия расслаивания растворов и осложняются существованием множества отсутствующих в макросистемах метастабильных состояний. Следствием может быть конкуренция сосуществующих растворов за место в core фазе, приводящая к би- или мультистабильности фазовых равновесий.

Актуальность описания размерных эффектов для трехкомпонентных растворов не ограничивается проблемами катализа. Подобные задачи возникают при создании и эксплуатации полупроводниковых материалов, ультрамелкозернистых сплавов, полимерных композиций, фармацевтических препаратов.

\section{Финансирование работы}

Работа выполнена в рамках государственного задания ИМХ РАН.

\section{Конфликт интересов}

Автор заявляет об отсутствии конфликта интересов.

\section{Список литературы}

[1] T. Tanaka. Mater. Sci. Forum 653, 55 (2010).

[2] G. Guisbiers, R. Mendoza-Pérez, L. Bazán-Díaz, R. MendozaCruz, J.J. Velázquez-Salazar, M. José-Yacamán. J. Phys. Chem. C 121, 6930 (2017).

[3] В.Б. Федосеев, Е.Н. Федосеева. ЖФХ 88, 3, 446 (2014).

[4] В.Б. Федосеев. ФТТ 57, 3, 585 (2015).

[5] F.H. Kaatz, A. Bultheel. Nanotechnology 29, 34, 345701 (2018).

[6] В.Б. Федосеев. Письма в ЖТФ 47, 3, 34 (2021).

[7] O. Kubaschewski, J.F. Counsell. Monatshefte Chem. 102, 6, 1724 (1971).

[8] A. Damjanovic, V. Brusić. Electrochim. Acta 12, 9, 1171 (1967).

[9] M.B. Gawande, A. Goswami, T. Asefa, H. Guo, A.V. Biradar, D.-L. Peng, R. Zboril, R.S. Varma. Chem. Soc. Rev. 44, 26, 7540 (2015).

[10] V. Peneau, Q. He, G. Shaw, S.A. Kondrat, T.E. Davies, P. Miedziak, M. Forde, N. Dimitratos, C.J. Kiely, G.J. Hutchings. Phys. Chem. Chem. Phys. 15, 26, 10636 (2013). L.B. Venarusso, J. Bettini, G. Maia. J. Solid State Electrochem. 20, 6, 1753 (2016).

[12] V. Tripkovic, H.A. Hansen, J. Rossmeisl, T. Vegge. Phys. Chem. Chem. Phys. 17, 17, 11647 (2015).

[13] D. Basu, S. Basu. Int. J. Hydrogen Energy 37, 5, 4678 (2012).

[14] L. Kuai, X. Yu, S. Wang, Y. Sang, B. Geng. Langmuir 28, 18, 7168 (2012).

[15] D. Chen, C. Li, H. Liu, F. Ye, J. Yang. Sci. Rep. 5, 19, 1 (2015).

[16] W. Xing, S.A. Kube, A.R. Kalidindi, D. Amram, J. Schroers, C.A. Schuh. Materialia 8, 100449 (2019).

[17] J. Li, H.M. Yin, X.B. Li, E. Okunishi, Y.L. Shen, J. He, Z.K. Tang, W.X. Wang, E. Yücelen, C. Li, Y. Gong, L. Gu, S. Miao, L.M. Liu, J. Luo, Y. Ding. Nature Energy 2, 8, 1 (2017).

[18] А.В. Шишулин, В.Б. Федосеев. Кинетика и катализ 60, 3, 334 (2019)

[19] Г.В. Белов, Г.Ф. Воронин, В.И. Горячева, А.Л. Емелина, И.А. Успенская. Математическое моделирование 18, 1, 67 (2006).

[20] G. Kaptay. J. Mater. Sci. 47, 24, 8320 (2012).

[21] А.Л. Восков. Расчет фазовых равновесий методом выпуклых оболочек. Дис. МГУ (2010).

[22] В.Б. Федосеев, А.В. Шишулин. ФТТ 60, 7, 1382 (2018).

[23] B.J. Keene. Int. Mater. Rev. 38, 4, 157 (1993).

[24] P. Qiao, S. Xu, D. Zhang, R. Li, S. Zou, J. Liu, W. Yi, J. Li, J. Fan. Chem. Commun. 50, 79, 11713 (2014).

Редактор Ю.Э. Китаев 